\title{
O impacto das condições bucais na qualidade de vida de pessoas com cinquenta ou mais anos de vida
}

\author{
The impact on life quality due to oral conditions \\ in people fifty years or above
}

Vinicius Cappo Bianco ${ }^{1}$ Eymar Sampaio Lopes $^{1}$ M aria H elena Borgato ${ }^{1}$ Poliana M oura e Silva ${ }^{1}$ Sara Nader $M$ arta ${ }^{1}$
${ }^{1}$ U niversidade do Sagrado Coração. Rual rmã Arminda 10-50, Jardim Brasil. 17011-160 Bauru SP. viniciusbianco79@hotmail.com
Abstract The objective of this work was to evaluate the influence of socio- demographic variables and oral health conditions in the quality of life of non-institutionalized people aged 50 or above, living in Bauru, São Paulo State. Clinical oral examinations were conducted on a sample of 224 people including dental caries, periodontal disease and usage or need of dental prosthesis according to W H O suggestions for community dental health surveys. They were also asked to answer a questionnaire comprised of 49 questions about the impact of their oral status on the activities of daily living (OHIP). Descriptive statistics and multiple regression analysis were computed with individual scores of each domain as dependent variables. The results were: $60 \%$ were re tired people, $42 \%$ exercise no social activity and $65.5 \%$ earned less than three minimum wages. Dentate people had a mean of 15.3 missing teeth, 117 people ( $53 \%$ of the sample) had full dentures. It could be concluded that regression analysis showed no socio-demographic variable but age having a significant impact on quality of life; as people get older oral disorders seems to produce lower impact on quality of life. As it concerns to oral clinical data only periodontal di sease showed impact on quality of life.

Key words Quality of life, Oral health, A ged people, Elderly
Resumo Avaliou-se a influência de variáveis sociodemográficas e as condições bucais na qualidade de vida de 224 indivíduos, com 50 anos ou mais de Bauru (SP). Os dados foram coletados de acordo com o Oral Health Surveys-Basic Methods: índices de cárie, periodontal comunitário, perda de inserção periodontal e uso/necessidade de próteses odontológicas. A percepção do indivíduo sobre suas condições bucais, foi medida pelo Oral Health Impact Profile, com sete domínios: limitação funcional, dor, desconforto psicológico, incapacidades física, psicológica e social e desvantagem. $60 \%$ dosindivíduoseram aposentados; $42 \%$ sem atividade social; $65,5 \%$ com renda mensal de um a três salários mínimos; $53 \%$ usavam prótese total superior e, destes, 33\% ambas; 1,94 dentes cariados e 5,4 restaurados; OH IP-os impactos sociais foram menos intensos que os físicos/ psicológicos; a idade foi significante em todos os domínios; apenas a condição periodontal foi importante para a qualidade de vida. As variáveis sociodemográficas e as condições bucais tiveram pequena variação no O HIP; os impactos sociais foram menos intensos que os físicos/psicológicos na qualidade de vida; a maioria dos entrevistados acusou pelo menos um impacto da saúde bucal na qualidade de vida; os escores do OHIP diminuíram nos grupos de maior idade.

Palavras-chave Qualidadedevida, Saúde bucal, Idosos 
Introdução

0 envelhecimento da população éum fenômeno mundial. Estima-seque, no ano de 2050, a população com mais de sessenta anos será de dois bilhões de habitantes ${ }^{1}$.

No Brasil, de 1940 a 2000, a população com idade acima de sessenta anos dobrou e a expectativa de vida subiu para 72,6 anos para mulhe res e 64,8 anos para os homens, contra uma mé dia de 50,9 anos na década de cinquenta 2 .

Esse fato, denominado transição demográfica e epidemiológica, é na verdade resultado da diminuição da natalidade, aumento da população economicamente ativa, diminuição das doenças infecciosas e aumento das doenças crônicodegenerativas ${ }^{3}$, como artrite, reumatismo, artrose, distúrbios cardiovasculares e diabetes ${ }^{4,5}$. Esta mudança dramática na composição etária da população indica a necessidade de elaboração de uma política de saúde planejada para atender a população mais idosa.

Além das doenças sistêmicas, a saúde bucal da maioria dos idosos no Brasil é precária, grande parte usa próteses, o número de dentes presentes na cavidade bucal épequeno e a incidência de cáriee doença periodontal éalta, fazendo com que problemas sociais, físicos e psicológicos afetem diretamente sua qualidade de vida $a^{6,7}$.

No entanto, a simples avaliação das condições clínicas não responde às questões: como 0 próprio paciente percebe a sua condição bucal e qual o efeito das más condições bucais sobre a sua qualidade de vida?

Muitos sistemas foram elaborados para responder essas questões; entre eles, temos o O ral $\mathrm{H}$ ealth Impact Profile $(\mathrm{OHIP})^{8}$ e o $\mathrm{GOHAI}^{9}$, que se utilizam de questionários respondidos pelo próprio paciente e indicam através de escores 0 grau do impacto da saúde bucal em sua qualidade de vida, contemplando aspectos físicos, sociais e psicológicos.

Assim, a proposta desse estudo foi de avaliar como os problemas de saúde bucal, mediados por condições socioeconômicas, em um grupo de pessoas com mais de cinquenta anos, afetam a qualidade devida, conhecimento esteque pode ser útil na definição e planejamento de programas preventivos e edu cati vos relacionados à saúde deste grupo da população que está em contínuo crescimento.

\section{M etodologia}

Participaram deste estudo 224 pessoas de ambos os sexos, com cinquenta anos ou mais, residentes no município de Bauru, pacientes das clínicas da Universidade do Sagrado Coração (USC) ou grupos deterceira idade. Foram excluídos da pesquisa indivíduos com problemas auditivos ou outra limitação física ou mental que afetasse sua capacidade de responder ao questionário. Aquel es que concordaram em participar do estudo assinaram previamenteo termo deconsentimento esclarecido aprovado pelo Comitê de Ética em Pesquisa da USC. Assim, incluiu-se $85 \%$ dos pacientes das clínicas da Universidade do Sagrado Coração (USC) ou grupos de terceira idade que se enquadravam nos critérios de inclusão acima descritos.

A razão de incluir pessoas com mais de cinquenta anos de idade foi para evitar o efeito floor, que se dá pelo grande número de escores zero nos exames ou na entrevista, como ocorre no caso dos desdentados totais, fazendo com que 0 índice perca a capacidade de discriminação ${ }^{10}$.

\section{Método}

Um formulário foi desenvolvido para o registro dasinformações pessoais quanto à idade, sexo, grau deescolaridade, ocupação, atividades sociais etempo decorrido desde a última visita ao dentista. Os dados dos exames foram coletados conforme as instruções do WH O-Oral Health Surveys-Basic $M$ ethods ${ }^{11}$ e incluíram avaliação da condição dentária (índiceCPO-D), índice periodontal comunitário (IPC), perda de inserção periodontal (PIP)), registrando-se também o uso e a necessidade de próteses odontológicas parciais ou totais.

Os exames clínicos foram realizados por um único examinador com auxílio de espelho e sonda periodontal (modelo Organização Mundial da Saúde), devidamente esterilizados. A consistência da avaliação bucal foi medida pelo exame clínico repetido nos primeiros 22 participantes ( $10 \%$ da amostra), respeitando-se um intervalo de tempo de sete a catorze dias e submetendo os resultados das duas avaliações ao teste Kappa. 0 resultado do Kappa sobre os exames repetidos apresentou um grau elevado de concordância, quevariou de 0,90 a 0,94 , sen do 0,94 para o CPOD; 0,93 para o IPC e 0,90 para o PIP. As entrevistas e os exames foram realizados em locais apropriados e, ocorrendo alguma condição dos tecidos moles ou dentais que indicasse necessidade de tratamento, foi dada orientação para exame 
mais detalhado ou eventual atendimento nas clínicas da USC.

Em seguida, os participantes receberam o questionário do OHIP8, composto por 49 questões, com a finalidadeavaliar o impacto produzido pela condição bucal na qualidade de vida. Essas questões são divididas em sete domínios: limitação funcional, dor física, desconforto psicológico, incapacidadefísica, incapacidadepsicológica, incapacidade social e desvantagem. As perguntas foram respondidas em uma escala de Likert com os seguintes escores: nunca $=0$; quase nunca $=1$; às vezes $=2$; frequentemente $=3$; muito frequentemente $=4$.

\section{Análisedos dados}

A análise dos dados incluiu o cálculo de estatísticas descritivas como média, desvio padrão, porcentagens e determinação de correlações. 0 coeficiente alfa de Cronbach foi calculado para comparar a consistência das respostas do questionário traduzido e aplicado na amostra, com os valores relatados por Slade eSpencer ${ }^{8}$. A análise de regressão múltipla foi realizada com o objetivo de determinar quais condições bucais esocioculturais produzem maior impacto na qualidade de vida. 0 nível escolhido para esse teste foi de $5 \%$ e o nível escolhido para exclusão foi de 10\%. Para realizar essa análise, as variáveis independentes não numéricas foram substituídas por valores numéricos apropriados. D as variáveis relativas à condição clínica bucal, descartou-se o CPO-D, optando-se por usar número de dentes ausentes. Para o restante dos exames (PIP, IPC, uso e necessidade de prótese), foram mantidos os mesmos escores da ficha de levantamento já citada.

\section{Resultados}

Características demográficas

e sociais da amostra

Dos 224 participantes, $158(70,53 \%)$ eram do sexo feminino e $66(29,46 \%)$, do sexo masculino, na faixa etária de cinquenta a oitenta anos ou mais de idade. No quesito escolaridade, verificou-se que a maioria $170(75,8 \%)$ tinha cursado até o primeiro ou segundo grau (Tabela 1). Com relação à aposentadoria, 137 (61,1\%) participantes se declararam aposentados. Quanto à ocupação, 101 (45,1\%) afirmaram ter algum tipo de trabalho (costureira, cabeleireira, motorista). No quesito atividades sociais, $98(43,7 \%)$ declara- ram pertencer a grupos de terceira idade, 25 $(11,2 \%)$ participavam de atividades religiosas e apenas $6(2,67 \%)$ eram engajados em clubes e praticavam esportes.

No item referente à renda, $149(66,5 \%)$ dos participantes recebiam de um a três salários mínimos, $42(18,8 \%)$ recebiam de quatro a sete salários mínimos e $33(14,7 \%)$ recebiam oito ou mais salários mínimos.

\section{Condições bucais dos indivíduos}

Os dados relativos ao uso ou a necessidade de algum tipo de prótese estão apresentados na Tabela 2. Verificou-se que 117 participantes (52\%) eram usuários de prótese total superior e, desses,

Tabela 1. Distribuição da amostra segundo a faixa etária, sexo e escolaridade.

\begin{tabular}{|c|c|c|c|c|}
\hline & \multicolumn{2}{|c|}{ Homens } & \multicolumn{2}{|c|}{ Mulheres } \\
\hline & $\mathrm{N}$ & $\%$ & $\mathrm{~N}$ & $\%$ \\
\hline \multicolumn{5}{|l|}{ Idade (anos) } \\
\hline 50 a 59 & 20 & 30,3 & 50 & 31,6 \\
\hline 60 a 69 & 22 & 33,3 & 55 & 34,8 \\
\hline 70 a79 & 21 & 31,8 & 43 & 27,2 \\
\hline 80 ou mais & 3 & 4,5 & 10 & 6,3 \\
\hline \multicolumn{5}{|l|}{ Escolaridade } \\
\hline Analfabeto & 3 & 4,6 & 9 & 5,7 \\
\hline Primeiro grau & 29 & 43,9 & 86 & 54,5 \\
\hline Segundo grau & 21 & 31,8 & 34 & 21,5 \\
\hline Universidade & 13 & 19,7 & 29 & 18,3 \\
\hline
\end{tabular}

Tabela 2. Distribuição percentual da amostra segundo o uso ou necessidade de prótese e arcos dentários envolvidos.

\begin{tabular}{|c|c|c|c|c|}
\hline & \multicolumn{2}{|c|}{ Superior } & \multicolumn{2}{|c|}{ Inferior } \\
\hline & $\mathrm{N}$ & $\%$ & $\mathrm{~N}$ & $\%$ \\
\hline \multicolumn{5}{|l|}{ Uso de prótese } \\
\hline Nenhuma & 36 & 16,1 & 72 & 32,1 \\
\hline Prótese fixa & 31 & 13,8 & 27 & 12,1 \\
\hline M ais de 1 prótese fixa & 10 & 4,5 & 12 & 5,4 \\
\hline Prótese parcial & 20 & 12,9 & 33 & 14,7 \\
\hline removível & 1 & 0,5 & 3 & 1,3 \\
\hline $\begin{array}{l}\text { Prótese fixa e removível } \\
\text { Prótese total }\end{array}$ & 117 & 52,2 & 77 & 34,4 \\
\hline \multicolumn{5}{|l|}{ N ecessidade de prótese } \\
\hline Nenhuma & 111 & 49,5 & 95 & 42,4 \\
\hline Prótese unitária & 6 & 2,7 & 5 & 2,2 \\
\hline M últiplos elementos & 26 & 11,6 & 52 & 23,2 \\
\hline Unitária e múltipla & 6 & 2,7 & 6 & 2,7 \\
\hline Prótese total & 75 & 33,5 & 66 & 29,5 \\
\hline
\end{tabular}


74 (33\%) também usavam prótese total inferior. No entanto, das pessoas portadoras de prótese total, $64(54,7 \%)$ necessitavam substituí-las.

Os IPC e PIP foram baseados nos exames de $129(57,6 \%)$ pessoas, pois foram excluídos os desdentados totais e os casos em que os dentes presentes eram insuficientes para a anotação. O escore 0 (normal) predominou tanto para o IPC quanto para o PIP. Mas, na somatória, a maioria dos examinados foram incluídos nos escores 1 (sangramento para IPC e perda de inserção de 4 a $5 \mathrm{~mm}$ para o PIP), 2 (cálculo para o IPC e perda de inserção de 6 a $8 \mathrm{~mm}$ para o PIP) e 3 (bolsa de 4 a $5 \mathrm{~mm}$ para IPC e perda de inserção de 9 a $11 \mathrm{~mm}$ para o PIP), atingindo $136(60,9 \%)$ e $125(55,8 \%)$ no IPC e PIP, respectivamente (Tabela 3 ). Ainda que 134 pessoas $(60 \%)$ tenham visitado o dentista há menos de dois anos, cerca de 45 (20\%) há dez anos não o faziam.

\section{Resultados do OHIP}

Os resultados do OHIP mostraram que os impactos sociais são menos intensos que os impactos físicos e psicológicos. 0 número de pessoas acusando al gum tipo de impacto em limitação funcional foi 203 (90,63\%), em dor física, 187 (83,48\%), incapacidadefísica, 164 (73,22\%). No domínio desconforto psicológico, os resultados foram $158(70,54 \%)$ e, em incapacidade psicológica, 120 (53,57\%). 0 contingentedepessoas acusando impactos em incapacidade social foi $55(24,55 \%)$ e, em desvantagem, $60(26,79 \%)$; portanto, muito menos que nos outros domínios. Os valores obtidos após a aplicação do co-

Tabela 3. Distribuição da amostra (129 indivíduos) segundo os valores do IPC e PIP.

\begin{tabular}{lrr}
\hline & $\mathrm{N}$ & \multicolumn{1}{c}{$\%$} \\
\hline Escore IPC & & \\
0: Normal & 53 & 41,1 \\
1: Sangramento & 8 & 6,2 \\
2: Cálculo & 32 & 24,8 \\
3: Bolsa 4-5mm & 27 & 29,9 \\
4: Bolsa 6+mm & 9 & 6,0 \\
Escore PIP & & \\
0: 0-3 mm & 57 & 44,2 \\
1: $4-5 \mathrm{~mm}$ & 36 & 27,9 \\
2: $6-8 \mathrm{~mm}$ & 29 & 22,5 \\
3: $9-11 \mathrm{~mm}$ & 7 & 5,4 \\
4: $12+\mathrm{mm}$ & - & 0,0 \\
\hline
\end{tabular}

eficiente de consistência de Cronbach em cada domínio foram: limitação funcional 0,67; dor física 0,61; desconforto psicológico 0,80; incapacidade física 0,71; incapacidade psicológica 0,74; incapacidade social 0,71; desvantagem 0,65.

A regressão múltipla foi realizada entre os sete domínios (variáveis dependentes) e as condições idade, sexo, escolaridade, aposentadoria, renda, atividade social, ocupação mais as condições bucaisnúmero de dentes ausentes, uso ou necessidade de prótesesuperior einferior, M ax-IPC e M axPIP (variáveis independentes), cujos resultadosestão apresentados na Tabela 4. A variável idade foi um fator significante em todos os domínios, com sinal negativo, indicando que, com o aumento da idade, os impactos diminuíam. A variável MaxIPC aparececomo significantenos domínios limitação funcional, dor física, incapacidade física e desvantagem, enquanto a variável número dedentes ausentes teve impacto significante para limitação funcional e incapacidade física. As variáveis ocupação, atividade social e PIP não apresentaram impacto em nenhum dos domínios.

Apresentou-se uma diminuição dos escores médios em direção dos grupos de maior idade, com exceção do domínio limitação funcional, ou seja, como já di to, nas pessoas mais idosas a saúde bucal tem menor impacto em sua qualidade de vida (Figura 1).

\section{Discussão}

Cabe iniciar a discussão com algumas considerações sobre a amostra e o processo de amostragem utilizado. Embora não se trate de um estudo epidemiológico, os dados obtidos fornecem informações interessantes sobre as condições sociais e de saúde bucal do grupo estudado, que, no entanto, não podem ser generalizadas, pois não existiu tentativa alguma de fazer-se uma amostragem casual. $\mathrm{Na}$ verdade, são raros os estudos epidemiológicos sobre saúde dental do idoso no Brasil. Colussi e Freitas ${ }^{12}$ encontraram apenas 31 publicações nacionais sobre saúde bucal do idoso no período de 1988 a 2001 e apenas oito de natureza epidemiológica; porém, somente uma utilizou amostragem probabilística. $\mathrm{Na}$ prática, as dificuldades para se obter amostragem de idosos leva os pesquisadores a utilizar amostras de grupos de terceira idade ou de clínicas para idosos. A amostra deste trabalho foi obtida dessa forma, mas é relativamente mais jovem que as utilizadas pela maioria dos estudos sobre idosos no Brasil, com exceção dos traba- 
Tabela 4. Regressão M últipla - sete domínios do OHIP e variáveis significantes.

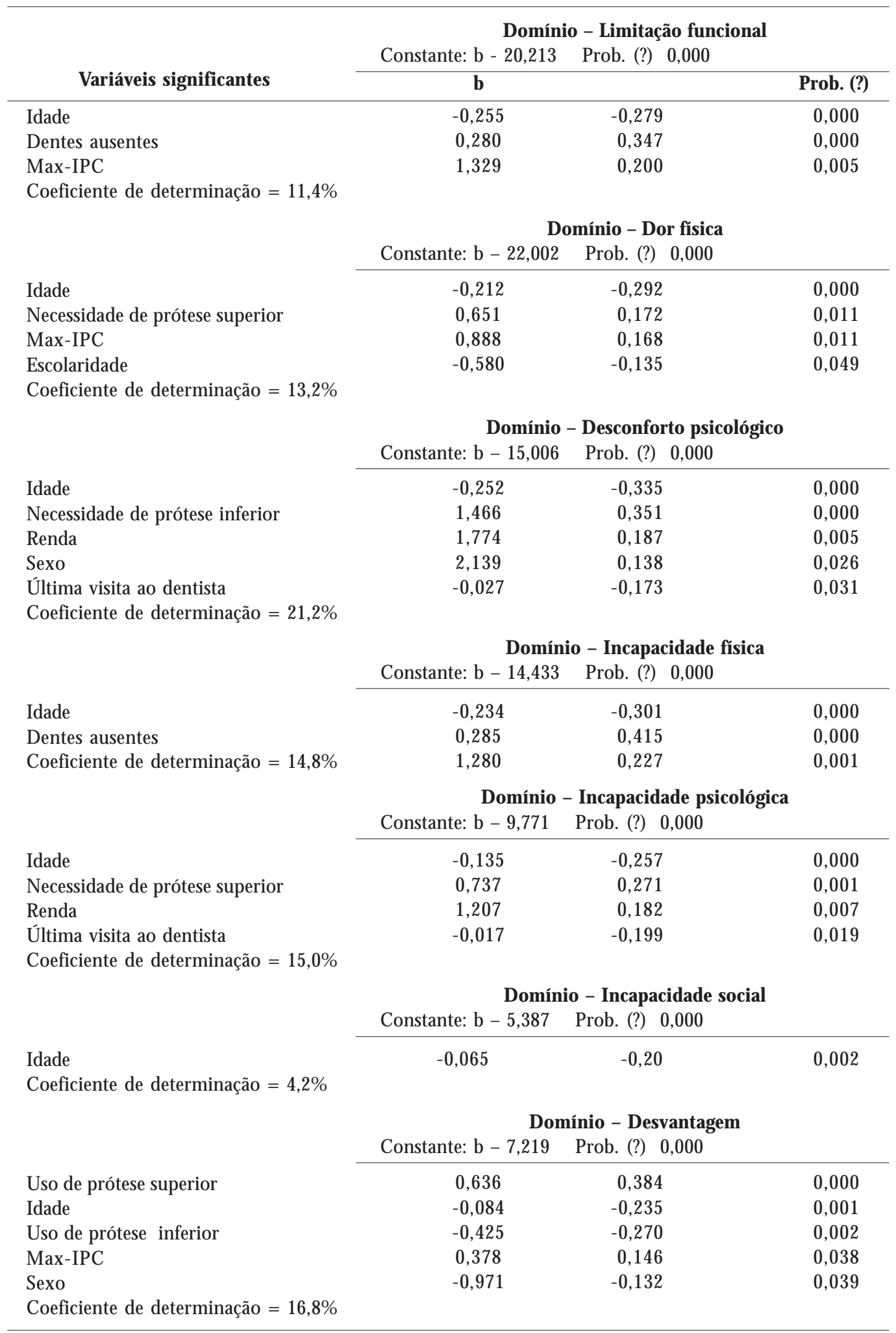

Ihos de Pereira et al. ${ }^{13}$, Saliba et al. ${ }^{14}$ eFrareet al. ${ }^{15}$. Quanto ao aspecto educacional, a amostra desta pesquisa está acima da média, com quase nenhum analfabeto e aproximadamente $50 \%$ com 


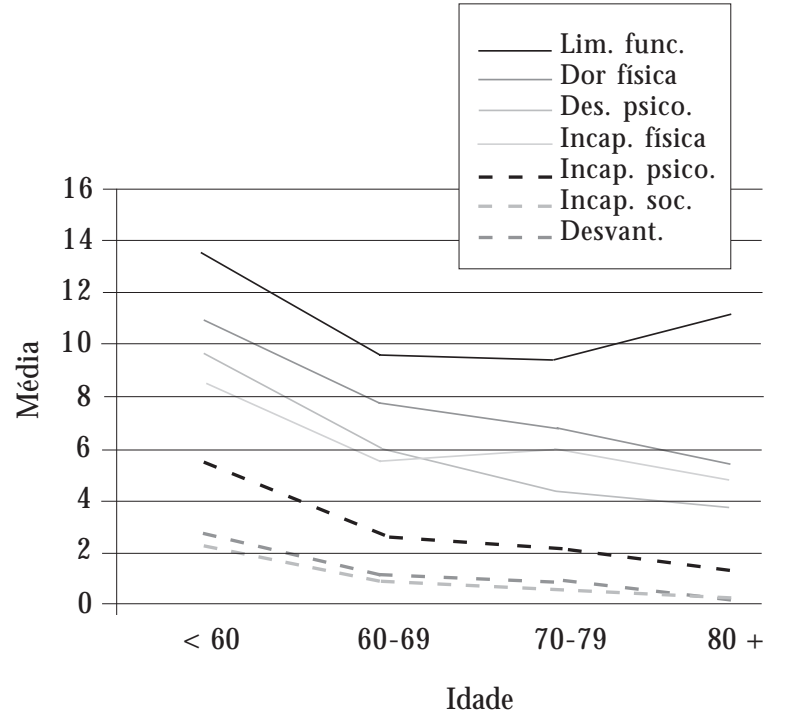

Figura 1. Ecores médios dos domínios do OHIP segundo a faixa etária. partir dos cinquenta anos deidade, $36 \%$ dos participantes eram edêntulos. Um dado adicional interessante foi que mais da metade das próteses totais foram registradas como necessitando de substituição.

0 alto índice deedentulismo foi também evidenciado nos resultados de Moreira et al. ${ }^{16}$ que realizaram uma revisão sistemática da literatura, no período de 1986 a 2004, para conhecer os problemas bucais mais prevalentes entre os idosos brasileiros e os principais obstáculos no acesso aos serviços de saúde. As principais barreiras para o acesso aos serviços odontológicos foram a baixa escolaridade, a baixa renda e a escassa oferta de serviços públicos de atenção à saúde bucal.

Um dos problemas na utilização dos índices de qualidade de vida está nas variações decorrentes de diferenças socioeconômicas e culturais. É difícil traduzir as questões originais de forma que tenham exatamente a mesma interpretação. Assim, a composição original do questionário pode ter sido prejudicada por uma compreensão diferente das questões. Essa deve ser a causa provável da diferença dos resultados do coeficiente de consistência deCronbach dessetrabalho $(0,61 \mathrm{a} 0,80)$, quando comparados aos val ores obtidos por Slade eSpencer ${ }^{8}$, com variação de 0,37 a 0,83.

Um outro aspecto a considerar nesteestudo é a dificuldade de análise por causa da presença de portadores de próteses totais superior e/ou inferior. De fato, os indicadores tradicionais de saúde bucal, baseados na contagem de cárie, restaurações, bolsa periodontal, cálculo e mesmo o conhecido CPO-D,etc., deixam de fazer sentido quando o número de dentes ausentes é muito grande. No caso deste trabalho, optou-se por incluir apenas o número de dentes ausentes, para evitar a dificuldade de interpretação associada às variáveis acima citadas. N esta decisão, levou-se em conta que, anteriormente, Locker ${ }^{17}$ já havia observado que apenas o número de dentes ausentes e a perda de ligamento periodontal acusavam impacto significante na apreciação subjetiva da condição bucal, não sendo significantes o número de coroas cariadas nem o número de raízes cariadas. $\mathrm{Na}$ prática, não havendo um indicador desaúde bucal queleveem conta as diferenças entre portadores e não portadores de prótese, a interpretação dos resultados deve ser cuidadosa e, se possível, deve-se separar os resultados em subgrupos. A elaboração de questionários diferentes para pessoas edêntulas e dentadas é importante, pois os quesitos do questionário usado apresentaram diferença de escores médios nas respostas do OHIP. 
Outro aspecto que merece destaquenesta discussão da percepção da qualidade de vida pelo idoso foi constatado no estudo de Cormack et al. ${ }^{18}$, no qual verificaram que a ausência de uma expectativa de vida pode levar a uma percepção dequalidade de saúde deficientee, mesmo diante de condições adversas de saúde geral e bucal, a maioria dos seus entrevistados permaneceram tendo uma percepção positiva a respeito de sua qualidade de vida, não havendo uma relação direta entre os dados clínicos encontrados e a percepção da qualidade de vida.

Quanto às variáveis sociodemográficas ocupação e atividade social, elas não apareceram como significantes em nenhum dos domínios. Leão e Sheiham ${ }^{19}$ avaliaram a importância do sexo e da classe social no escore DIDL, obtendo significância estatística apenas para classe social $\left(R^{2}=0,03\right)$, quando o modelo de regressão incluía apenas estas duas variáveis; porém, deixou de ser significante quando as variáveis referentes à saúde bucal (dentais e periodontais) foram incluídas. Da mesma forma, Corrêa da Silva eFernandes ${ }^{9}$ não conseguiram identificar participação significante das variáveis independentes idade, sexo, escolaridade e classe social no modelo final de regressão. Nesta pesquisa, as variáveis sociodemográficas parecem estar associadas aos domínios psicológicos (sexo, renda eúltima visita apareceram em desconforto psicológico e, novamente, renda eúltima visita mais o sexo em incapacidade psicológica). Curiosamente, escolaridade apareceu como marginalmente significante em dor física.

Os dados coletados mostraram que as medidas geralmente utilizadas para avaliar clinicamente a saúde bucal têm pouca correlação com os impactos que as alterações bucais produzem na qualidade de vida das pessoas, além de ser de difícil manejo estatístico se utilizados em uma ampla faixa de idade, porque cada uma delas avalia uma dimensão diferente, como presença de cárie, dentes ausentes e sem opositores, inflamação periodontal, higi ene bucal, lesões de mucosa, articulação temporomandibular, etc., difíceis de integrar deuma forma lógica numaúnica medida de saúde bucal. Por outro lado, é necessário identificar melhor as variáveis demográficas e sociais que, interagindo com os aspectos clínicos, produzem impactos na qualidade de vida. Ainda, a saúde oral foi percebida com pouca importância entre os idosos, que apesar de valorizarem muito o paladar e a manutenção da dentição permanente, consideraram a perda como al go natural econsequente da idade ecomo uma condição passível de reposição com as próteses dentárias, como identificaram Somchinda et al. ${ }^{20}$.

Estes dados podem ser explorados em outros aspectos, procurando detalhar as interrelações entre essas variáveis, buscando melhorar o entendimento num ambiente multidimensional complexo como este.

0 fato de que pouco da variação total foi explicado pelo modelo de regressão utilizado é um indício de que ainda há muito que se aprender sobre como os problemas de saúde afetam a qualidade de vida.

\section{Conclusões}

As variáveis sociodemográficas e as condições bucais aqui analisadas explicaram apenas pequena porcentagem da variação dos impactos à saúde medidos pelo OHIP. Os dados indicaram que os impactos sociais são menos intensos que os físicos e psicológi cos na qualidade de vida.

A pesar de os impactos serem, de maneira geral, baixos, grande parte dos entrevistados acusou pelo menos um impacto da saúde bucal na qualidade de vida. Os escores médios do OHIP tenderam a diminuir em direção dos grupos de maior idade. 


\section{Colaboradores}

V Cappo Bianco desenvolveu a parteexperimental e a redação do artigo; ES Lopes foi o orientador do trabalho, responsável pelo delineamento do experimento eanálise einterpretação dos dados; $\mathrm{MH}$ Borgato colaborou na organização dos dados e na redação; PM Silva auxiliou na coleta de dados e tabulação dos mesmos; SN Marta trabalhou na revisão crítica e na concepção da redação final.

\section{Referências}

1. United Nations. Report of the Second World Assembly on Ageing. Madrid: UN; 2002. p. 8-12.

2. Pessini L. Envelhecimento e saúde: ecos da II Assembléia M undial sobre o envelhecimento. M undo saúde 2002; 26:457-463.

3. Pereira AC, Silva FRB, M eneghim MC. Prevalência de cárie e necessidade de prótese em uma população geriátrica institucionalizada da cidade de Piracicaba. ROBRAC 1999; 8:17-21.

4. Oliveira JA, Ribeiro EDP, Bonachela WC, Cappelozza ALA. Perfil do paciente odontogeriátrico da faculdade de odontologia de Bauru-USP. Rev Bras Protese Clin Lab 2002; 4:71-79.

5. Pivetta M. Retratos do envelhecer. Revista Pesquisa FAPESP 2003; 87:32-39.

6. Corrêa da Silva SR, Fernandes RAC. Auto percepção das condições de saúde bucal por idosos. Rev. Saude Publica 2001; 35:349-355.

7. Silva PB. Saúde bucal e qualidade de vida de um grupo de idosos de Araçatuba-SP: U tilização do Oral Health Impact Profile (OHIP-14), e caracterização do perfil sócio-econômico dos entrevistados [dissertação]. Araçatuba (SP): UNESP; 2000.

8. Slade GD, Spencer AJ. Development and evaluation of the Oral Health Impact Profile. Community Dent Health 1994; 11: 3-11.

9. Atchison KA, Dolan TA. Development of the geriatric oral health assessment index. J Dent Educ. 1990; 54:680-687.

10. Locker D, Allen PF. Developing short form measures of oral health - related quality of life. J Public Health Dentist. 2002; 62:13-20.

11. World Health Organization. Oral Health Surveys Basic M ethod. $4^{\text {th }}$ ed. Geneva: WH 0; 1987. p. 26-29.

12. Colussi CF, Freitas SFT. Aspectos epidemiológicos da saúde bucal dos idosos no Brasil. Cad Saude Publica 2002; 18:1313-1320.
13. Pereira M G. Epidemiologia teórica e prática. Rio de Janeiro: Guanabara Koogan;1996.

14. Saliba CA, Saliba NA, M arcelino G, M oimaz SAS. Auto avaliação de saúde na terceira idade : sobre saúde bucal através de um inquérito. Rev Gaucha Odontol 1999; 47:127-130.

15. Frare SM, Limas PA, Albarello EJ, Pedot G, Régio RAS. Terceira idade: Quais os problemas bucais existentes? Rev Assoc Paul Cir Dent 1997; 51:573-576.

16. Moreira RS, Nico LS, Tomita NE, Ruiz T. A saúde bucal do idoso brasileiro: revisão sistemática sobre o quadro epidemiológico e acesso aos serviços de saúde bucal. Cad Saude Publica 2005; 21(6):1665-1675.

17. Locker D. The burden of oral disorders in a population of older adults. Community Dent $\mathrm{H}$ ealth 1992; 9:109-124.

18. Cormack EF, Gevaerd S, Schmidt C, Reichow R. Saúde bucal e qualidade de vida do idoso. [site da Internet] [acessado 2008 out 11]. Disponível em: http://www.odontologia.com.br/artigos. asp?id=284 \&idesp $=19 \&$ ler $=s$

19. Leão A, Sheiham A. The development of sociodental measure of dental impact of daily living. Community Dental Health 1996; 13:22-26.

20. Somchinda A, Chamoschine FF. Saúde e qualidade de vida na terceira idade: uma introspecção dos idosos institucionalizados [monografia]. Brasília (DF)I: ABO; 2003.

Artigo apresentado em 19/05/2008

Aprovado em 25/08/2008

Versão final apresentada em 11/10/2008 